\title{
Cofactores Ambientales Asociados al Desarrollo de Neoplasia Intraepitelial Cervical y Cáncer Cervicouterino
}

\section{Environmental Cofactors Associated with the Development of Cervical Intraepithelial Neoplasia and Cervical Cancer}

\author{
Medina-de la Cruz Omar,2, Villegas-Hinojosa Edgar ${ }^{1}$, Ruíz-Baca Estela ${ }^{3}$, Gallegos-García Verónica ${ }^{1 *}$ \\ 1. Facultad de Enfermería y Nutrición. Universidad Autónoma de San Luis Potosí. \\ 2. Hospital General de Zona No. 50. Instituto Mexicano del Seguro Social. \\ 3. Facultad de Ciencias Químicas. Universidad Juárez del Estado de Durango.
}

\begin{abstract}
*Autor de correspondencia: Gallegos-García Verónica
Facultad de Enfermería y Nutrición, Universidad Autónoma de San Luis Potosí, San Luis Potosí, México.

Dirección: Av. Niño Artillero No. 130. Zona Universitaria. C.P. 78240. veronica.gallegos@uaslp.mx
\end{abstract}

DOI http://dx.doi.org/10.28960/revmeduas.2007-8013.v12.n1.008

Recibido 30 de marzo 2021, aceptado 28 de julio 2021

RESUMEN

El Cáncer Cervicouterino ( $\mathrm{CaCu}$ ) es una de las principales causas de morbimortalidad a nivel mundial y en México. Esta enfermedad requiere de la presencia del Virus del Papiloma Humano (VPH) para su progreso a Neoplasia Intraepitelial Cervical (NIC), pero además de otros cofactores. Entre estos se han propuesto los cofactores ambientales o exógenos, los de tipo viral, del huésped o intrínsecos y los de tipo social. Esta revisión aborda los de tipo ambiental, entre ellos están: el uso de anticonceptivos hormonales orales, hábito tabáquico, exposición al humo de biomasa, la dieta, trauma cervical, Inicio de Vida sexual a Edad Temprana (IVSET), múltiples parejas sexuales, coinfección con otras enfermedades de transmisión sexual, no utilización del preservativo y la circuncisión de la pareja. Los cofactores modulan el progreso de los distintos grados de NIC a CaCu, por ello es importantes conocerlos y analizar de qué manera influyen en la aparición esta patología.

Palabras Clave: Virus del Papiloma Humano, Cáncer Cervicouterino, Cofactores ambientales

\section{ABSTRACT}

Cervical Cancer (CC) is one of the main causes of morbidity and mortality worldwide and in Mexico. This disease requires the presence of the Human Papilloma Virus (HPV) for its progress to Cervical Intraepithelial Neoplasia (CIN), but in addition to other co-factors. Among these, environmental or exogenous cofactors, those of the viral, host or intrinsic and those of the social type have been proposed. This review addresses those of an environmental nature, among them are: the use of oral hormonal contraceptives, smoking, exposure to biomass smoke, diet, cervical trauma, Early Sexual Life Onset (IVSET), multiple sexual partners, co-infection with other sexually transmitted diseases, non-use of condoms and circumcision of the couple. The co-factors modulate the progress of the different grades of CIN to CC, therefore it is important to know them and analyze how the influence the appearance of this pathology. Keywords: Human Papillomavirus, Cervical Cancer, Environmental Co-factors.

\section{Introducción}

Actualmente las enfermedades no transmisibles son responsables de la mayoría de muertes a nivel mundial, una de las principales causas es el cáncer y se considera una barrera para aumentar la esperanza de vida en el siglo XXI ${ }^{1}$. La incidencia y la mortalidad por cáncer ha aumentado, las razones son complejas como los cambios en la prevalencia y la distribución de cofactores para el desarrollo de neoplasias ${ }^{2}$.
De acuerdo a Global Observatory Cancer (GLOBOCAN) en el 2018 hubo 569,847 nuevos casos de Cáncer Cervicouterino $(\mathrm{CaCu})$ y 311,365 defunciones a nivel mundial ${ }^{1}$. En México en ese año se diagnosticaron 7,869 nuevos casos y 4,121 muertes, siendo la segunda causa de muerte por cáncer en mujeres solo después del cáncer de mama ${ }^{3}$.

Antes de progresar a $\mathrm{CaCu}$ la zona de transformación del cérvix pasa por diferentes cambios que van desde una infección por VPH hasta el 
desarrollo de una Neoplasia Intraepitelial Cervical (NIC I, NIC II y NIC III) de acuerdo a la clasificación de Richart ${ }^{4}$. Existen otros cofactores importantes que se encargan de modular la infección por VPH e influyen en el riesgo de progresión ${ }^{5}$. Estos se pueden clasificar en tres grupos; 1) cofactores ambientales o exógenos, 2) cofactores virales, 3) cofactores del huésped o intrínsecos, 4) cofactores sociales ${ }^{5-11}$, en esta revisión nos centraremos en describir los cofactores ambientales.

\section{Cofactores ambientales o exógenos}

Las diferencias geográficas en la distribución del cáncer pueden deberse en parte a la variación humana individual, en la activación y desintoxicación de carcinógenos ambientales, a continuación, se abordan algunos de los cofactores ambientales importantes para el desarrollo de NIC ${ }^{12}$.

\section{Anticonceptivos orales}

La Zona de Transformación (ZT) del cuello uterino en donde el VPH inicia una NIC, depende de hormonas esteroides como la progesterona que influye sobre el efecto que ocasiona el VPH en las células epiteliales cervicales ${ }^{13}$. El uso de Anticonceptivos Orales (AO) como cofactor no está bien establecido, la International Agency Research of Cancer (IARC) clasificó a los anticonceptivos hormonales combinados de estrógeno-progestina y la terapia de reemplazo hormonal como cancerígenos para los humanos ${ }^{14}$.
Un estudio multicéntrico realizado por la IARC encontró que el uso de AO por menos de cinco años no tiene relación con el desarrollo de $\mathrm{CaCu}$, aunque el riesgo incremento casi tres veces más la probabilidad para las pacientes que lo usaron entre 5-9 años más y las mujeres que tomaron $\mathrm{AO}$ por más de 10 años tenían cuatro veces más la probabilidad de desarrollar $\mathrm{CaCu}$ respecto a las que no ${ }^{15}$.

Las hormonas sexuales se relacionan como cofactores que favorecen los efectos celulares de la infección por VPH y existen varios mecanismos por los cuales ocurre esto. Uno de ellos menciona que los $\mathrm{AO}$ contienen estradiol y progesterona, estas hormonas se unen y activan los receptores de estrógenos (ER $\alpha$ y ER $\beta$ ) y progesterona (PR-A y PR-B) respectivamente activando factores de transcripción dependientes de ligando que podrían estar jugando un papel clave en la progresión y desarrollo de $\mathrm{CaCu}$ 16.

Otro mecanismo menciona que la ausencia de hormonas como el estrógeno en el receptor $E R \alpha$ y ER $\beta$ es inactivo y se localiza en el núcleo de la célula blanco como un gran complejo asociado a proteínas de choque térmico. Sin embargo, cuando la hormona se une a su receptor, se produce un cambio conformacional que provoca la dimerización del receptor adquiriendo la capacidad de unión a secuencias específicas reguladoras de la transcripción del Ácido Desoxirribonucleico (ADN) del VPH ${ }^{17,18}$. 
La progesterona estimula la integración del ADN del VPH-16 en el genoma del huésped, de esta manera aumenta la expresión de las oncoproteínas E6/E7 del VPH que actúan en la regulación del ciclo celular y la apoptosis degradando genes supresores de tumores como p53 y de esta manera se induce la carcinogénesis ${ }^{6}$. En el 2014 se comparó el transcriptoma de pacientes con $\mathrm{CaCu}$ que utilizaron o no $\mathrm{AO}$ para identificar la Expresión Diferencial de Genes (EDG), se identificaron 80 genes y se confirmó que el proceso metabólico se enriqueció en la EDG en pacientes con $\mathrm{CaCu}{ }^{19}$.

\section{Hábito tabáquico}

La exposición a carcinógenos como los del humo del tabaco, se han sugerido como un posible cofactor promotor de $\mathrm{CaCu}{ }^{12}$. El humo del tabaco contiene y libera más de 6,000 compuestos, algunos de los cuales son agentes carcinógenos que ya se conocen como: bencil (a) pirenos, Hidrocarburos Aromáticos Policíclicos (HAPs) y nitrosaminas específicas del tabaco. Uno de los principales compuestos es el 4-(metilnitrosamino)-1-(3-piridil)-1-butanona o NNK ${ }^{20,}$ 21.

El moco cervical de fumadoras contiene tres veces los niveles de NKK respecto a las no fumadoras, el mecanismo de transporte es que llegan a las células epiteliales cervicales a través de la sangre ${ }^{20}$. Los carcinógenos del cigarrillo crean aductos en el ADN y se han detectado al- gunos compuestos sobre células epiteliales cervicales, esto sugiere un papel potencial de tales carcinógenos en la patogénesis de $\mathrm{CaCu}{ }^{12}$.

En investigaciones en líneas celulares cervicales con genomas episomales de VPH expuestos al humo de tabaco se observó mayor expresión del oncogén E6 y E7, esto sugiere un papel importante en la modulación del VPH en el desarrollo de $\mathrm{CaCu}{ }^{22}$. Baltazar-Rodríguez en su estudio en mujeres con $\mathrm{CaCu}$ considera el hábito tabáquico como el haber consumido más de 100 cigarrillos durante la vida ${ }^{23}$.

\section{Exposición al humo de biomasa}

La literatura científica al menos en Latinoamérica indica que la inhalación de aire contaminado a través de la combustión de carbón, madera (como la leña, ya que aún existe esta práctica para la cocción de los alimentos) o paja se asocia con la incidencia de enfermedades como cáncer de pulmón, del tracto digestivo superior; en el CaCu la información es controversial ${ }^{12}$. Durante la combustión de leña se liberan HAPs (considerados mutagénicos y cancerígenos) como benzopirenos, dibenzoantracenos y dibenzocarbazoles; otros productos que también se liberan son aldehídos, fenoles, cresoles, quinonas, óoxidos de nitrógeno y azufre, hidrocarburos alifáticos y monóxido de carbono ${ }^{24}$. Estos productos han sido considerados por la IARC como posibles cancerígenos en humanos ${ }^{12}$.

En Honduras un estudio en pacientes con $\mathrm{CaCu}$ encontró que el número de años en donde las 
mujeres estuvieron expuestas al humo de biomasa fue mayor en los casos respecto a los controles, el Odds Ratio (OR) fue 3.67 en mujeres con 25-34 años de exposición al humo, mientras que las que tenían más de 35 años expuestas el OR fue de $6.35^{7}$. La exposición por varios años puede tener un efecto biológico en la progresión de las lesiones pre-neoplásicas del epitelio cervical, induciendo la acumulación de daño genético a manera de aberraciones cromosómicas y mutaciones genéticas, como se ha propuesto para otros carcinógenos ambientales, favoreciendo así la aparición de NIC en mujeres positivas a VPH ${ }^{21}$.

\section{Dieta}

El estilo de vida es una forma de proporcionar, mantener y mejorar la salud, por lo tanto, el cáncer puede prevenirse mediante la modificación de hábitos alimenticios ${ }^{25}$. Una alimentación saludable que incluya el consumo de frutas, verduras y la ingesta de algunos nutrientes (antioxidantes, folatos y minerales) se asocia a una disminución del riesgo de infección por VPH y su progreso a NIC ${ }^{8}$. La relación entre la carcinogénesis cervical y la dieta no están completamente descritas, aunque la presencia de algunos micronutrientes como carotenoides, tocoferoles, vitaminas y minerales modifican el progreso de la infección por VPH a CaCu ${ }^{26}$.

En el 2010 se demostró que frutas y verduras que contienen vitamina $\mathrm{C}$ y retinol contribuyen a disminuir la carga viral de VPH y el progreso a NIC ${ }^{27}$. La vitamina C es participa en reacciones de hidroxilación en donde se involucra el oxígeno molecular, nitratos, inhibe la carcinogénesis y reduce el daño en los cromosomas de las células ${ }^{28}$.

González-Acevedo y cols. demostraron que las mujeres con mayor ingesta de vitamina $C$ y de Zinc presentan menor riesgo para desarrollar $\mathrm{CaCu}{ }^{29}$. La vitamina $\mathrm{C}$ podría estar actuando como eliminador de radicales libres y oxidantes, ya que si no se pueden neutralizar la inflamación causada por VPH puede provocar daño en el ADN 30,31 .

El Zinc es una línea de defensa de las células contra especies reactivas de oxígeno para eliminar el anión superóxido, además la asociación entre la deficiencia de Zinc y el cáncer ha sido descrita debido a que juega un papel indispensable en la protección del daño en el ADN por su actividad antioxidante y es un componente que participa en los mecanismos de reparación del ADN 29.

Entre los antioxidantes dietéticos que pueden actuar en diferentes etapas de la historia natural del $\mathrm{CaCu}$ se encuentran la vitamina $\mathrm{A}$, la vitamina $D$ y la papaya, estos pueden ser más efectivos para prevenir la displasia temprana (NIC I) [31]. En contraste la vitamina E y el licopeno, pueden tener más efecto para prevenir la displasia tardía (NIC II y NIC III), otros alimentos como las verduras contribuyen a prevenir la infección por VPH, la NIC I-III y el CaCu ${ }^{31,32}$. Las 
vitaminas $A, C, D$ y $E$ contribuyen en la inhibición de células tumorales, pueden estabilizar la proteína p53, prevenir el daño del ADN celular y reducir la inmunosupresión ${ }^{31}$.

En el año 2012 se realizó un metanálisis y los resultados indican que la ingesta de vitamina $A$ esta asociada con un riesgo reducido por $\mathrm{CaCu}$. El consumo de retinol $(\mathrm{OR}=0.80)$ tiene una asociación débil para el riesgo de $\mathrm{CaCu}$, pero el consumo de caroteno $(\mathrm{OR}=0.51)$ y carotenoides $(0 \mathrm{R}=0.48)$ tienen una fuerte asociación inversa con el riesgo de cáncer ${ }^{33}$.

Las vitaminas protegen a las células de los efectos secundarios de especies reactivas de oxígeno; la vitamina A puede actuar en la división y diferenciación celular, regula el sistema inmunitario, previene el desarrollo de células cancerosas, proporciona propiedades antimutagénicas, antitumorales, inmunoestimulantes, antiulcerosas y degenerativas en el humano ${ }^{34}$. Los carotenoides y los tocoferoles tienen actividades antioxidantes y efectos moduladores sobre la inmunidad, aunque deben realizarse más estudios para un mejor entendimiento de como los alimentos tienen este efecto ${ }^{35}$.

\section{Trauma cervical}

Los incidentes que pueden ocasionar traumatismos en el cuello uterino, se consideran cofactores de riesgo para el desarrollo de $\mathrm{CaCu}$. Las mujeres que se encuentran más susceptibles a esto son las que han sufrido laceraciones en el cérvix, mutilación genital, pacientes que han tenido partos por vía vaginal, aquellas con antecedentes de episiotomía, por mencionar algunas causas ${ }^{10}$.

En mujeres de Sudan se encontró que aquellas que tuvieron algún tipo de trauma cervical eran aproximadamente nueve veces más propensas al $\mathrm{CaCu}$ en comparación con las mujeres que no lo tuvieron ${ }^{10}$. Las mujeres con cesárea mostraron riesgo reducido para el desarrollo de neoplasia respecto a las pacientes que tenían partos vaginales ${ }^{36}$.

Los cambios metaplásicos que se desarrollan por el trauma cervical durante el trabajo de parto, se han identificado como riesgo mayor para aquellas mujeres que son multíparas ${ }^{36}$. El riesgo de $\mathrm{CaCu}$ se incrementó en más de dos veces en aquellas pacientes que tuvieron más de cuatro hijos respecto a las que solo tenían uno o ninguno ${ }^{37,38}$.

Panjaliya menciona que el trauma del cérvix durante el parto deja la ZT expuesta lo que facilita una infección por VPH, además de que puede haber otras causas para una mayor susceptibilidad a infección como la inmunosupresión, la influencia de las hormonas y la dieta ${ }^{39}$. La episiotomía, la laceración cervical y la mutilación genital son los principales tipos de traumas cervicales iatrogénicos ${ }^{40}$.

\section{Inicio de vida sexual a edad temprana}

El CaCu se desarrolla en la ZT, esta es la unión entre el epitelio columnar de una sola capa y el 
epitelio escamoso estratificado distal, creando de esta manera la unión escamo-columnar. Durante la adolescencia esta ZT está activa y se encuentra evertida hacia el exocérvix quedando más expuesta y de esta forma durante el coito el VPH puede llegar e integrarse en las células epiteliales ${ }^{41}$.

En una investigación del 2008 argumentan que las mujeres que inician su vida sexual antes de los 20 años son más susceptibles a contraer una infección por VPH ${ }^{23}$. Durante la pubertad los tejidos cervicovaginales son más susceptibles ante agentes cancerígenos ya que la ZT es más proliferativa, además con la posibilidad de tener múltiples compañeros sexuales y mayor riesgo de exposición al VPH ${ }^{42}$.

Otro estudio realizado en el estado de Zacatecas observó que el riesgo de NIC es mayor cuando el IVSET, en pacientes que iniciaron su vida sexual antes de los 15 años tuvieron un riesgo de dos veces mayor respecto a las que iniciaron después de los 20 años ${ }^{43}$.

\section{Múltiples parejas sexuales}

Diferentes estudios epidemiológicos muestran que el comportamiento sexual es un factor determinante para adquirir una infección por VPH, en aquellos con múltiples parejas sexuales ${ }^{44}$. Cuanto mayor sea el número de parejas sexuales que una mujer tenga sin el uso de preservativo, mayor será el riesgo de entrar en contacto con el VPH ${ }^{45}$.
En un estudio de casos y controles realizado por Kashyap menciona un $\mathrm{OR}=5$ para el desarrollo de $\mathrm{CaCu}$ en pacientes con más de tres parejas sexuales respecto a las que solo tuvieron una 11. Se ha sugerido que la mayoría de infecciones por VPH de alto riesgo sucede en mujeres jóvenes, estas suelen ser transitorias e involucran contactos sexuales con nuevas parejas, sin embargo, pueden persistir en algunas mujeres ${ }^{46}$.

Se ha propuesto que la actividad sexual de mujeres solteras tiende a ser de manera esporádica, aunque se ha observado que existe una creciente prevalencia del sexo fuera del matrimonio 47,48 . Los hombres juegan un rol importante en el aumento del riesgo para que sus parejas desarrollen $\mathrm{CaCu}$, sin dejar de lado que un mayor número de parejas sexuales aumenta el riesgo de infectarse por $\mathrm{VPH}^{49}$.

Un factor de confusión importante es el número de parejas sexuales que haya tenido durante su vida la pareja actual, ya que en los estudios no hay datos sobre el patrón de comportamiento sexual de los hombres $u$ otros datos relevantes acerca de ello ${ }^{50}$. Un estudio en España indica que la presencia de ADN del VPH en el pene del esposo aumenta en cinco veces más el riesgo de sus esposas para desarrollar $\mathrm{CaCu}{ }^{51}$. El comportamiento sexual puede tener más variables, sin embargo, debido al sesgo de deseabilidad social, la tendencia en los participantes de los estudios puede responder a las expectativas sociales de lo que es aceptable, algunas 
encuestas han encontrado que las mujeres tienden a subestimar el número de parejas sexuales ${ }^{52}$. Por lo tanto, se deben realizar más investigaciones que permitan conocer más sobre esto y su asociación con el desarrollo de $\mathrm{CaCu}$.

\section{Coinfección con otras enfermedades de} transmisión sexual

Las mujeres entre los 15-24 años tienen tasas más altas de infecciones de transmisión sexual, entre las que se incluye Chlamydia trachomatis, Neisseria gonorrhoeae, Trichomona vaginalis y VPH ${ }^{53}$. Estos pueden ocasionar inflamación local y pueden contribuir al VPH y la progresión de la lesión cervical ${ }^{54}$.

Se sabe que las células epiteliales columnares son objetivos para la infección por $C$. trachomatis y $N$. gonorrehoeae, mientras que la zona de transformación es ideal para la infección por VPH ya que requiere de la replicación de la célula huésped y la diferenciación para su replicación ${ }^{53}$. Amorim detectó $T$. vaginalis en el $61.3 \%$ de mujeres con algún grado de NIC, por lo tanto, hubo mayor riesgo relacionado con la presencia de este microorganismo y el desarrollo de lesiones cervicales ${ }^{54}$.

La respuesta inflamatoria por los microorganismos en el sitio de infección induce al daño celular que sugiere lesiones cervicales ${ }^{54}$. C. trachomatis infecta las células epiteliales del tracto genital, de esta manera produce inflamación y está implicada en el riesgo de NIC ${ }^{55}$. Un estudio de cohorte con un seguimiento de 19 años mostró que la infección repetida por $C$. trachomatis aumenta el riesgo de NIC entre las mujeres con una infección persistente por VPH de alto riesgo 56 .

El rol que juega una ETS como cofactor de VPH es la de facilitar la entrada y la persistencia del VPH por la inflamación cervical crónica, ulceración del epitelio cervical y reducción de la inmunidad $^{57}$. La investigación de Abreu y cols. indico que la coinfección de VPH y C. trachomatis exhibió cinco veces más el riesgo de una NIC ${ }^{58}$.

\section{No utilización de métodos de barrera}

La prevención primaria basada en métodos anticonceptivos de barrera como el preservativo (condón) y la circuncisión son métodos eficientes, aunque por la población hay muy poca inclinación para aceptar este tipo de métodos. Para lo cual es importante difundir información adecuada para disminuir ETS ${ }^{59}$.

El condón confiere protección contra el VPH de diferentes maneras: 1) su uso reduce la infección por VPH y la reinfección, así el sistema inmunitario repara la lesión cervical; 2) el semen tiene un efecto inmunosupresor, esto es una ventaja en el proceso reproductivo pero cuando existen lesiones cervicales, la ausencia de semen ayuda a la respuesta inmune celular; 3) el látex de los condones es un material ajeno al cuerpo que puede desencadenar la respuesta inmune celular y contribuir a la regresión de una lesión ${ }^{60}$. 
En el 2008 la Food and Drug Administration (FDA) cambio el etiquetado de los condones y los reconoce útiles para prevenir la infección por VPH y otras ETS ${ }^{61}$. Winer menciona que las mujeres cuyas parejas usaron condones el $100 \%$ del tiempo durante 8 meses anteriores redujeron las probabilidades de contraer el VPH respecto aquellas cuyas parejas usaron condones menos del $5 \%$ de las veces ${ }^{62}$. Se debe tomar en cuenta que pueden existe errores en la colocación del condón, problemas como roturas, deslizamientos o aplicación tardía son de las más comunes; lo anterior no se toma en cuenta al momento de realizar los estudios ${ }^{63}$.

\section{Circuncisión masculina}

Cuando la circuncisión masculina (CM) se realiza en la infancia ofrece diferentes beneficios, como la protección contra infecciones del tracto urinario ${ }^{64}$. Se ha demostrado la vulnerabilidad del prepucio a diferentes ETS como el virus de inmunodeficiencia humana $(\mathrm{VIH})$, ya que la cavidad del prepucio tiene un gran microbioma y la superficie de la mucosa contiene células diana para agentes infecciosos 65 .

Las mujeres con parejas con CM tienen menos probabilidad de adquirir ETS, también se asocia a un menor riesgo del desarrollo de $\mathrm{CaCu}$, de NIC, infección por el virus del herpes simple tipo 2 , clamidia y sífilis ${ }^{66}$. Se han comparado la prevalencia de VPH en hombres con y sin circuncisión, en diferentes países, razas y edades ${ }^{9}$. Un estudio de New England Journal of Medicine en el 2002 encontró que el VPH se detectó en $19.6 \%$ en 847 hombres sin CM respecto al 5.5\% que corresponde a 292 hombres que la tenían 67. Otro estudio realizado en Dinamarca menciona que estar sin CM se asocia a una probabilidad de cinco veces más de tener una infección por $\mathrm{VPH}{ }^{68}$.

En México una investigación en hombres que acudieron a realizarse la vasectomía tenían un riesgo de cinco veces más de tener una infección por VPH si no se les había realizado la circuncisión ${ }^{69}$. Otro estudio en el que participaron militares sanos identificó un OR 10 veces mayor a la infección por VPH en aquellos sin circuncisión ${ }^{70}$.

Los hombres sin circuncisión tienen más posibilidad de infectarse por VPH, ya que en el coito el revestimiento interno del prepucio queda expuesto a secreciones vaginales. Después del coito, el inoculo de VPH queda atrapado en la cavidad del prepucio y se transmite a las células de la superficie a las capas basales, de esta manera se producen cambios en las células basoloides y el virus se puede replicar, así durante las siguientes relaciones sexuales se puede producir una infección en la ZT 65 .

La cavidad del prepucio tiene un ambiente húmedo, esto proporciona un ambiente favorable para la infección por VPH en comparación con los que tienen $\mathrm{CM}^{71}$. Otra explicación es que el VPH se replica en células epiteliales basales de la epidermis, sin embargo, después de la cir- 
cuncisión la queratinización de la cicatriz quirúrgica y el tejido circundante puede ayudar a re- ducir dicha infección ${ }^{9}$. En la Figura 1 se muestra un resumen de los cofactores mostrados en esta revisión.

Figura 1. Historia natural de la enfermedad de $\mathrm{CaCu}$. Progreso del epitelio cervical sano hasta el cáncer (1-6). Cofactores ambientales que contribuyen a la modulación de la infección por virus del papiloma humano (VPH) y su progreso a los distintos grados de NIC y CaCu. IVSET: Inicio de vida sexual a edad temprana. ETS: Enfermedad de transmisión sexual.

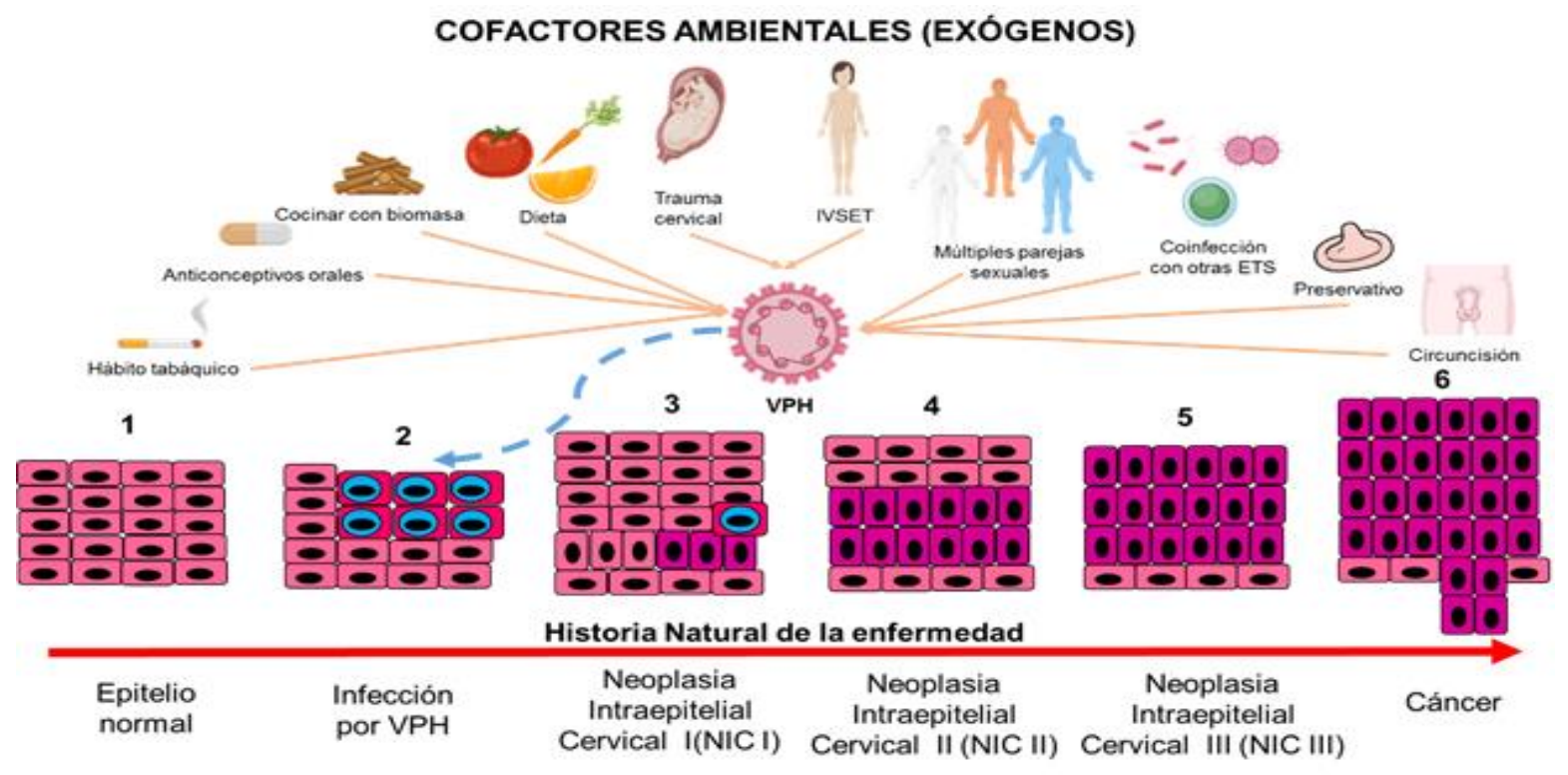

\section{Conclusión}

Los cofactores ambientales o exógenos dependen directamente de las mujeres, por lo tanto, son cofactores que pueden ser modificados y de esta manera contribuir a disminuir la persistencia de una infección por VPH y su progreso mediante educación para la salud y prevención primaria en los sistemas de salud y así evitar el desarrollo a una NIC que podría tener como consecuencia la evolución al $\mathrm{CaCu}$.

\section{Referencias}

1. Bray F, Ferlay J, Soerjomataram I, Siegel RL, Torre LA, Jemal A. Global cancer statistics 2018: GLOBOCAN estimates of incidence and mortality worldwide for 36 cancers in 185 countries. CA Cancer J Clin 2018;68(6):394-424 .

2. Lin L, Yan L, Liu Y, Yuan F, Li H, Ni J. Incidence and death in 29 cancer groups in 2017 and trend analysis from 1990 to 2017 from the Global Burden of Disease Study. J Hematol Oncol 2019;12(1):96.

3. World Health O. Global health Observatory, 2018. Geneva: health organization[Online], Available: who int/gho/database/en.

4. Hernández-Hernández DM, Apresa-García T, Patlán-Pérez RM. Epidemiological overview of uterine cervical cancer. Rev Med Inst Mex Seguro Soc. 2015;53(S2):154-61.

5. Castellsagué X, Munoz N. Chapter 3: Cofactors in human papillomavirus 
carcinogenesis-role of parity, oral contraceptives, and tobacco smoking. JNCl Monograp 2003;2003(31):20-8.

6. Loopik DL, IntHout J, Melchers WJG, Massuger LFAG, Bekkers RLM, Siebers AG. Oral contraceptive and intrauterine device use and the risk of cervical intraepithelial neoplasia grade III or worse: a populationbased study. Eur J Cancer 2020;124:102-9.

7. Ferrera A, Velema JP, Figueroa M, Bulnes $\mathrm{R}$, Toro LA, Claros JM, et al. Co-factors related to the causal relationship between human papillomavirus and invasive cervical cancer in Honduras. Int $\mathrm{J}$ Epidemiol 2000;29(5):817-25.

8. Barchitta M, Maugeri A, La Mastra C, Rosa MCL, Favara G, Lio RMS, et al. Dietary Antioxidant Intake and Human Papillomavirus Infection: Evidence from a Cross-Sectional Study in Italy. Nutrients 2020;12(5):1384.

9. Morris BJ, Gray RH, Castellsague X, Bosch FX, Halperin DT, Waskett JH, et al. The strong protective effect of circumcision against cancer of the penis. Adv Urol $2011 ; 2011$.

10. Ibrahim A, Rasch V, Pukkala E, Aro AR. Cervical cancer risk factors and feasibility of visual inspection with acetic acid screening in Sudan. Int J Women's Health 2011;3:117.

11. Kashyap N, Krishnan N, Kaur S, Ghai S. Risk Factors of Cervical Cancer: A CaseControl Study. Asia Pac J Oncol Nurs 2019;6(3):308.

12. Sierra-Torres $\mathrm{CH}$, Arboleda-Moreno $\mathrm{YY}$, Orejuela-Aristizabal L. Exposure to wood smoke, HPV infection, and genetic susceptibility for cervical neoplasia among women in Colombia. Environ Mol Mutagen 2006;47(7):553-61.

13. Chin HJ, Lee AH, Colville L, Xu D, Binns CW. Condom and oral contraceptive use and risk of cervical intraepithelial neoplasia in Australian women. J Gynecol Oncol 2014;25(3):183-7.

14. Grevers X, Grundy A, Poirier AE, Khandwala $F$, Feldman M, Friedenreich $\mathrm{CM}$, et al. Cancer incidence attributable to the use of oral contraceptives and hormone therapy in Alberta in 2012. CMAJ Open 2016;4(4):E754.

15. Moreno V, Bosch FX, Muñoz N, Meijer CJLM, Shah KV, Walboomers JMM, et al. Effect of oral contraceptives on risk of cervical cancer in women with human papillomavirus infection: the IARC multicentric case-control study. Lancet 2002;359(9312):1085-92.

16. Chung S-H. Targeting female hormone receptors as cervical cancer therapy. Trends Endocrinol Metab 2015;26(8):399-401.

17. Chung S-H, Franceschi S, Lambert PF. Estrogen and ERa: Culprits in cervical cancer?. Trends Endocrinol Metab 2010;21(8):504-11.

18. Shew ML, McGlennen R, Zaidi N, Westerheim $\mathrm{M}$, Ireland $\mathrm{M}$, Anderson $\mathrm{S}$. Oestrogen receptor transcripts associated with cervical human papillomavirus infection. Sex Transmit Infec 2002;78(3):210-4.

19. Gao T, Wang J, Yang M, Li H. Transcriptome analysis reveals the effect of oral 
contraceptive use on cervical cancer. Mol Med Rep 2014;10(4):1703-8.

20. Haverkos HW. Viruses, chemicals and cocarcinogenesis. Oncogene 2004;23(38):6492-9.

21. Haverkos HW, Haverkos GP, O’Mara M. Cocarcinogenesis: human papillomaviruses, coal tar derivatives, and squamous cell cervical cancer. Front Microbiol 2017;8:2253.

22. Wei L, Griego AM, Chu M, Ozbun MA. Tobacco exposure results in increased E6 and $E 7$ oncogene expression, DNA damage and mutation rates in cells maintaining episomal human papillomavirus 16 genomes. Carcinog 2014;35(10):2373-81.

23. Baltazar-Rodriguez LM, Anaya-Ventura A, Andrade-Soto $M$, Monrroy-Guizar $E A$, Bautista-Lam JR, Jonguitud-Olguin G, et al. Polymorphism in the matrix metalloproteinase-2 gene promoter is associated with cervical neoplasm risk in Mexican women. Bioch Genet 2008;46(34):137-44.

24. Morales Vargas R. Contaminación del aire y efectos tóxicos por partículas respirables (PM10) en el humo, de madera en comercios de alimentos San José-Costa Rica. Rev Cost Sal Púb 2003;12(22):16-28.

25. Yeganeh Z, Sheikhan Z, Kariman N, Esteki T, Dolatian M, Mahmoodi Z, et al. Lifestyle and nutritional risk in women with cervical cancer: A comparative study. Int J Fertil Womens Med 2019;7(4):501-7.

26. Medina de la Cruz O, Villegas-Hinojosa E, Gaytán-Hernández D, Gutiérrez-Enríquez
SO, Gallegos-García V. Identificación de cofactores nutricionales asociados a neoplasia intraepitelial cervical en mujeres de San Luis Potosí. Tequio 2018; 4 (1):3747.

27. Hwang JH, Lee JK, Kim TJ, Kim MK. The association between fruit and vegetable consumption and HPV viral load in high-risk HPV-positive women with cervical intraepithelial neoplasia. Cancer Causes Control 2010;21(1):51-9.

28. Labani L, Andallu B, Meera M, Asthana S, Satyanarayana L. Food consumption pattern in cervical carcinoma patients and controls. Indian J Med Paediatr Oncol 2009;30(2):71.

29. Acevedo OG, Ugalde PZ, García VG, de León Martínez LD, Hernández DG. Ingesta de antioxidantes y su asociación a Cáncer Cervicouterino en mujeres de un Sistema Universitario. RESPYN Rev Sal Pub Nut 2020;19(1):23-32.

30. Guo L, Zhu H, Lin C, Che J, Tian X, Han S, et al. Associations between antioxidant vitamins and the risk of invasive cervical cancer in Chinese women: A case-control study. Scient Rep 2015;5:13607.

31. Koshiyama $M$, Nakagawa $M$, Ono A. The Preventive Effect of Dietary Antioxidants Against Cervical Cancer Versus the Promotive Effect of Tobacco Smoking. Healthcare. 2019; 7: 162.

32. Koshiyama M. The effects of the dietary and nutrient intake on gynecologic cancers. Healthcare 2019; 7: 88.

33.Zhang X, Dai B, Zhang B, Wang Z. Vitamin $A$ and risk of cervical cancer: a meta- 
analysis. Gynecol Oncol 2012;124(2):36673.

34. Shrivastava A, Pradhan S, Mishra SP, Asthana AK, Choudhary S, Zahra K, et al. Serum vitamin $A, E$ and $C$ status in cervical cancer patients undergoing Concurrent Chemo-Radiotherapy, an institutional study. J Nutr Intermed Metab 2019;18:100107.

35. Fujii T, Takatsuka N, Nagata C, Matsumoto $\mathrm{K}$, Oki A, Furuta $\mathrm{R}$, et al. Association between carotenoids and outcome of cervical intraepithelial neoplasia: a prospective cohort study. Int J Clinic Oncol 2013;18(6):1091-101.

36. Muñoz N, Franceschi S, Bosetti C, Moreno V, Herrero R, Smith JS, et al. Role of parity and human papillomavirus in cervical cancer: the IARC multicentric case-control study. Lancet 2002;359(9312):1093-101.

37. Brinton LA, Reeves WC, Brenes MM, Herrero R, De BrilTon RC, Gaitan E, et al. Parity as a risk factor for cervical cancer. Ame J Epidemiol 1989;130(3):486-96.

38. Becker TM, Wheeler CM, McGough NS, Stidley CA, Parmenter CA, Dorin MH, et al. Contraceptive and reproductive risks for cervical dysplasia in southwestern Hispanic and non-Hispanic white women. Int $\mathrm{J}$ Epidemiol 1994;23(5):913-22.

39. Panjaliya R, Dogra V, Gupta S. Study of the risk factors associated with cervical cancer. Biomed Pharmacol J 2015;3(1):179-82.

40. Brady M. Female genital mutilation: complications and risk of HIV transmission. AIDS Patient Care and STDs 1999;13(12):709-16.
41. Hwang LY, Lieberman JA, Ma Y, Farhat S, Moscicki A-B. Cervical ectopy and the acquisition of human papillomavirus in adolescents and young women. Obstet Gynecol 2012;119(6):1164.

42. Serrano RO, Pérez CJU, Martínez LAD, Romero YRD. Factores de riesgo para cáncer de cuello uterino. Rev Colom Obstet Ginecol 2004;55(2):146-60.

43. Castañeda-Iñiguez MS, Toledo-Cisneros R, Aguilera-Delgadillo M. Factores de riesgo para cáncer cervicouterino en mujeres de Zacatecas. Salud Publica Mex 1998;40:3308.

44. Liu Z-C, Liu W-D, Liu Y-H, Ye X-H, Chen SD. Multiple sexual partners as a potential independent risk factor for cervical cancer: a meta-analysis of epidemiological studies. Asian Pac J Cancer Prev 2015;16(9):3893900.

45. Duarte DV, Vieira RC, de Brito EB, Pinheiro MdCN, Monteiro JdSV, Valente MDR, et al. Prevalence of human papillomavirus infection and cervical cancer screening among riverside women of the Brazilian amazon. Rev Bras Ginecol Obstet 2017;39(07):350-7.

46. Chan PKS, Chang AR, Cheung JLK, Chan DPC, Xu LY, Tang NLS, et al. Determinants of cervical human papillomavirus infection: differences between high-and lowoncogenic risk types. J Infec Dis 2002;185(1):28-35.

47. Wellings K, Collumbien M, Slaymaker E, Singh S, Hodges Z, Patel D, et al. Sexual 
behaviour in context: a global perspective. Lancet 2006;368(9548):1706-28.

48. Nnko S, Boerma JT, Urassa M, Mwaluko G, Zaba B. Secretive females or swaggering males?: An assessment of the quality of sexual partnership reporting in rural Tanzania. Social Sci Med 2004;59(2):299310.

49. Lenselink CH, Melchers WJG, Quint WGV, Hoebers AMJ, Hendriks JCM, Massuger LFAG, et al. Sexual behaviour and HPV infections in 18 to 29 year old women in the pre-vaccine era in the Netherlands. PLoS One 2008;3(11).

50. International Collaboration of Epidemiological Studies of Cervical C. Cervical carcinoma and sexual behavior: collaborative reanalysis of individual data on 15,461 women with cervical carcinoma and 29,164 women without cervical carcinoma from 21 epidemiological studies. Cancer Epidemiol Biomarkers Prev 2009;18(4):1060.

51. Castellsagué X, Bosch FX, Muñoz N. The male role in cervical cancer. Salud Pub Mex 2003;45:345-53.

52. Roik E, Sharashova E, Kharkova O, Nieboer E, Postoev V, Odland JØ. Sociodemographic characteristics, sexual behaviour and knowledge about cervical cancer prevention as risk factors for high-risk human papillomavirus infection in Arkhangelsk, North-West Russia. Int J Circumpolar Health 2018;77(1):1498681.

53. Hwang LY, Ma Y, Benningfield SM, Clayton L, Hanson EN, Jay J, et al. Factors that influence the rate of epithelial maturation in the cervix in healthy young women. $J$ Adolescent Health 2009;44(2):103-10.

54. Amorim AT, Marques LM, Campos GB, Lobão TN, de Souza Lino V, Cintra RC, et al. Co-infection of sexually transmitted pathogens and Human Papillomavirus in cervical samples of women of Brazil. BMC Infect Dis 2017;17(1):769.

55.Zhu H, Shen Z, Luo H, Zhang W, Zhu X. Chlamydia trachomatis infection-associated risk of cervical cancer: a meta-analysis. Med 2016;95(13).

56. Jensen KE, Thomsen LT, Schmiedel S, Frederiksen $\mathrm{K}$, Norrild $\mathrm{B}$, van den Brule $\mathrm{A}$, et al. Chlamydia trachomatis and risk of cervical intraepithelial neoplasia grade 3 or worse in women with persistent human papillomavirus infection: a cohort study. Sex Transm Infect 2014;90(7):550-5.

57. Alotaibi HJ, Almajhdi FN, Alsaleh AN, Obeid $\mathrm{DA}$, Khayat $\mathrm{HH}$, Al-Muammer TA, et al. Association of Sexually Transmitted Infections and Human Papillomavirus CoInfection with Abnormal Cervical Cytology among Women in Saudi Arabia. Saudi J Biol Sci 2020.

58.de Abreu ALP, Malaguti N, Souza RP, Uchimura NS, Ferreira ÉC, Pereira MW, et al. Association of human papillomavirus, Neisseria gonorrhoeae and Chlamydia trachomatis co-infections on the risk of highgrade squamous intraepithelial cervical lesion. American journal of cancer research 2016;6(6):1371.

59. Ngoma M, Autier P. Cancer prevention: cervical cancer. Cancer Med Sci 2019;13. 
60. Skorstengaard M, Suhr J, Lynge E. Condom use to enhance regression of cervical intraepithelial neoplasia: study protocol for a randomized controlled trial. Trials 2019;20(1):473.

61. Tsai C-C, Wang Y-Y, Chou Y-H, Li C-C, Wu W-J. Update on strategies of controlling sexually transmitted infections: Taiwan experience. Urol Sci 2016;27(3):122-30.

62. Winer RL, Hughes JP, Feng Q, O'Reilly S, Kiviat NB, Holmes KK, et al. Condom use and the risk of genital human papillomavirus infection in young women. $\mathrm{N}$ Engl J Med 2006;354(25):2645-54.

63. Lam JUH, Rebolj M, Dugué P-A, Bonde J, von Euler-Chelpin M, Lynge E. Condom use in prevention of Human Papillomavirus infections and cervical neoplasia: systematic review of longitudinal studies. J Med Screen 2014;21(1):38-50.

64. Cathcart P, Nuttall Mv, Van der Meulen J, Emberton M, Kenny SE. Trends in paediatric circumcision and its complications in England between 1997 and 2003. Brit J Sur 2006;93(7):885-90.

65. Morris BJ, Hankins CA, Banerjee J, Lumbers ER, Mindel A, Klausner JD, et al. Does Male Circumcision Reduce Women's Risk of Sexually Transmitted Infections, Cervical Cancer, and Associated Conditions? Front Public Health 2019;7.

66. Grund JM, Bryant TS, Jackson I, Curran K, Bock N, Toledo $\mathrm{C}$, et al. Association between male circumcision and women's biomedical health outcomes: a systematic review. Lancet Glob Health 2017;5(11):e1113-e22.
67. Castellsagué X, Bosch FX, Munoz N, Meijer CJLM, Shah KV, De Sanjosé S, et al. Male circumcision, penile human papillomavirus infection, and cervical cancer in female partners. N Engl J Med 2002;346(15):110512.

68. Svare EI, Kjaer SK, Worm AM, Østerlind A, Meijer C, Van den Brule AJC. Risk factors for genital HPV DNA in men resemble those found in women: a study of male attendees at a Danish STD clinic. Sex Trans Infect 2002;78(3):215-8.

69. Vaccarella S, Lazcano-Ponce E, CastroGarduño JA, Cruz-Valdez A, Díaz V, Schiavon $R$, et al. Prevalence and determinants of human papillomavirus infection in men attending vasectomy clinics in Mexico. Int J Cancer 2006;119(8):1934-9.

70. Lajous M, Mueller N, Cruz-Valdéz A, Aguilar LV, Franceschi S, Hernández-Ávila M, et al. Determinants of prevalence, acquisition, and persistence of human papillomavirus in healthy Mexican military men. Cancer Epidemiol Biomarkers Prev 2005;14(7):1710-6.

71. Weiss HA, Thomas SL, Munabi SK, Hayes RJ. Male circumcision and risk of syphilis, chancroid, and genital herpes: a systematic review and meta-analysis. EN 2006;82(2):101-10. 